\title{
Anesthetic Management for Quadricuspid Aortic Valve Repair: Case Report and Literature Review
}

\author{
Lúcia Caroline Schons Correa ${ }^{1}$, Érica Mendonça Reiff Carlos ${ }^{1}$, \\ Paula Cristina Leitão de Assunção ${ }^{1}$, José Eduardo Guimaraes Pereira², \\ Carlos Darcy Alves Bersot ${ }^{1}$
}

\author{
${ }^{1}$ Department of Anesthesiology, Medical Sciences College at Hospital Universitário Pedro Ernesto (HUPE), Universidade do \\ Estado do Rio de Janeiro (UERJ), Rio de Janeiro, Brazil \\ ${ }^{2}$ Department of Anaesthesiology, Botucatu Medical School, Universidade Estadual Paulista, São Paulo, Botucatu, Brazil \\ Email: carlosbersot@gmail.com
}

How to cite this paper: Correa, L.C.S., Carlos, É.M.R., de Assunção, P.C.L., Pereira, J.E.G. and Bersot, C.D.A. (2018) Anesthetic Management for Quadricuspid Aortic Valve Repair: Case Report and Literature Review. Open Journal of Anesthesiology, 8, 172-182.

https://doi.org/10.4236/ojanes.2018.85018

Received: March 8, 2018

Accepted: May 28, 2108

Published: May 31, 2108

Copyright $\odot 2018$ by authors and Scientific Research Publishing Inc. This work is licensed under the Creative Commons Attribution International License (CC BY 4.0).

http://creativecommons.org/licenses/by/4.0/

\begin{abstract}
Quadricuspid aortic valve (QAV) is a rare congenital cardiac anomaly with prevalence less than $0.01 \%$ in autopsy series. It can be found as an isolated anomaly, and the patient may be asymptomatic, but is often associated with aortic insufficiency in $68 \%$ of the cases. Transthoracic echocardiography (TTE) and transesophageal echocardiography (TEE) are currently used as a diagnostic and follow-up method for this anomaly. The objective of the present paper is through the clinical case report of a 60 year old patient submitted to surgical correction of QAV, to relate some clinical aspects of the disease and to discuss the anesthetic management for this group of patients, in addition, emphasizing the importance and the increasing use of the TEE in the intraoperative period in cardiac surgeries.
\end{abstract}

\section{Keywords}

Quadricuspid Aortic Valve, Anesthetic Management, Transesophageal Echocardiography

\section{Introduction}

There are two classification systems for QAV: The Hurwitz \& Roberts [1], in which the QAV is classified according to the size of the supranumerary cusp into seven categories from A to G, and to which Vali [2] has added the $\mathrm{H}$ type (Figure 1). And the system proposed by Nakamura et al. [3], in which the classification is based on the position of the supranumerary cusp, whether it is located 
between the right and left coronary cusps (type 1), between the right and non-coronary cusps (type 2), between left and non-coronary cusps (type 3 ), and type 4 when there are four equal sized cusps present.

Functionally, the QAV often presents itself as pure aortic regurgitation (AR). Tutarel \& Westhoff-Bleck [4] reported that the functional status of QAV was regurgitant in $74.7 \%$, combined stenosis and regurgitation in $8.4 \%$, stenotic in $0.7 \%$, and normally functioning in $16.2 \%$.

QAV may occur in up to $32 \%$ of the cases in association with a variety of heart disorders like, atrial septal defect [5], ventricular septal defect [6], patent ductus arteriosus [7], tetralogy of Fallot [8], sinus of Valsalva fistula [9], subaortic fibromuscular stenosis [10], mitral valve regurgitation [11], mitral valve prolapse [12], hypertrophic non-obstructive cardiomyopathy (with echocardiographic evidence of massive left ventricular hypertrophy and asymmetric septal hypertrophy) [13], and transposition of the great arteries [14].

The early diagnosis of this pathology is of great importance due to the serious consequence that this can cause: severe aortic regurgitation, which may lead, in extreme cases, to left ventricular failure. In addition, there is a probable increase in the risk of developing infective endocarditis (IE) and possibility of association with anomalous origin of coronary artery ostia. Thus, patients with this congenital abnormality should have a permanent strict medical follow-up, since the diagnosis, and must undergo surgical correction (if indicated) avoiding evolution to left ventricular disease (ventricular failure) [15] [16].

TEE, in addition to its importance in the diagnosis of the disease and the follow-up of these patients, has been gaining great importance in its intraoperative use in cardiac surgeries due to the ease of real-time hemodynamic monitoring, analysis of anatomical structures and early identification of complications such as ischemia, air embolism or pericardial effusion [17] [18].

The surgical indications for QAV are severe AR [19], severe aortic stenosis [20], or dysfunctional QAV associated with other lesions, such as occlusion of the left coronary ostium [19]. The treatment for QAV consists on surgical correction, either by valve replacement or valve repair. Thus, the technique of choice for the procedure is usually determined on the disease severity, condition of QAV, and surgeon's preference [21]. Postoperative complications are not frequent, and they include progressive AR, transient ischemic attack, cardiac arrest and postoperative complete heart block [22]. The overall survival rates of QAV patients were $89.9 \%$ and $84.9 \%$ at 5- and 10 -year follow-up, respectively [19].

\section{Case Report}

A 60-year-old male patient has hypertensive, on regular use of simvastatin and amlodipine. Diagnosis of QAV was performed in 2017 when it was admitted to Cardiology department after the onset of dyspnea symptoms at medium effort. The TTE showed severe aortic insufficiency with dilation of the aorta root (4 
$\mathrm{cm}$ ) and ascending aorta $(5.2 \mathrm{~cm})$, ejection fraction of $49 \%$, enlargement of the left chambers, borderline left ventricle function and aortic reflux.

Coronary angiography is without severe obstructive lesions, with mild LV dysfunction. Laboratory tests within the limits of normality electrocardiogram with sinus rhythm and evidence of increased left chambers. Chest x-ray: Dilation of ascending aorta. Patient hospitalized in the Cardiology sector of the Pedro Ernesto University Hospital (HUPE) for clinical compensation and surgical correction. Surgical proposal: aortic valve replacement and ascending aortic prosthesis.

The patient was admitted to the surgical theater, decompensated, with blood pressure 140/70 $\mathrm{mmHg}$, SpO2: $88 \%$ in ambient air, heart rate of $90 \mathrm{bpm}$. After entry into the operating room, he was premedicated with intravenous midazolam $3 \mathrm{mg}$ and then pre-oxygenated under face mask. Punctured right radial artery for continuous invasive blood pressure monitoring, followed by anesthetic induction with etomidate $10 \mathrm{mg}$, fentanyl $200 \mathrm{mcg}$ and muscle relaxation with rocuronium $50 \mathrm{mg}$. Orotracheal intubation was performed with $8 \mathrm{~mm}$ cuff under direct laryngoscopy. Hypnosis was maintained with sevoflurane $1 \%-3 \%$. Then 2 g cefazolin and tranexamic acid (Transamin) 2.7 g were infused, puncturing right internal jugular vein with the aid of ultrasonography.

Before beginning the surgery, a TEE probe was introduced to monitor cardiac function and visualize structures of interest (Image 1). The patient was anticoagulated with 25,000 I.U. heparin, and the initial activated clotting time (ACT) of 890 seconds was used. During extracorporeal circulation (ECC), propofol was started in a continuous infusion pump to maintain hypnosis. The ECC lasted for

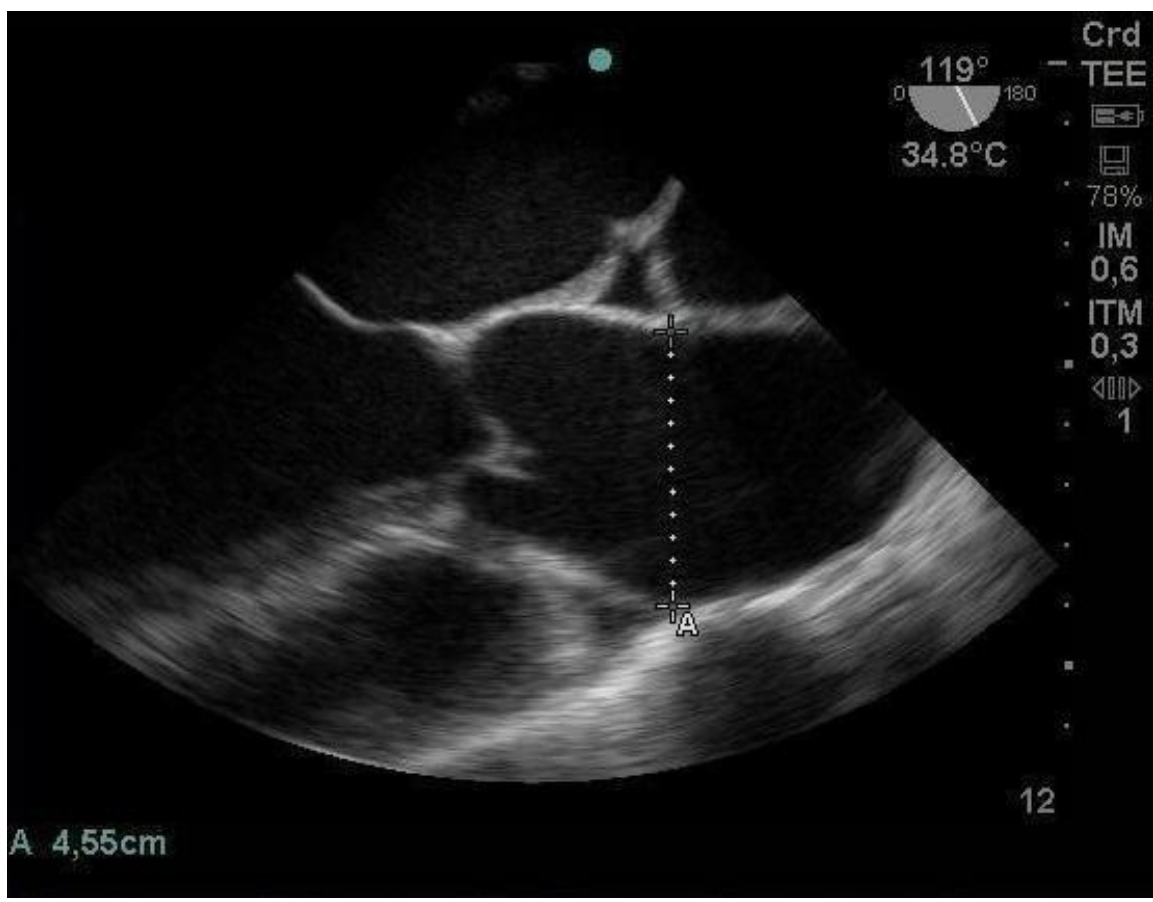

Image 1. Mid-esophageal cut short aortic valve obtained through intraoperative TEE exam, demonstrating dilation of the aortic root $(4.5 \mathrm{~cm})$. 
125 minutes and the aortic clamping of 100 minutes. After ECC, he was shocked 2 times by the surgeon for ventricular fibrillation. Infusion of noradrenaline and dobutamine were started, titrating according to the patient's response. During the hemostasis review, protamine $300 \mathrm{mg}$ was infused, with the final ACT of 88 seconds.

At the end of the surgery, the patient was transported to the intensive care unit, intubated, with assisted ventilation and infusion of noradrenaline at 0.15 $\mathrm{mcg} / \mathrm{kg} / \mathrm{min}$ and dobutamine $10 \mathrm{mcg} / \mathrm{kg} / \mathrm{min}$. In the postoperative period, the patient was extubated and evolved without pain complaints and good hemodynamic stability in the immediate postoperative period. The patient ambulatory segment evolves with functional capacity improvement and without complications in the late postoperative period. He presented new TTE without aortic insufficiency and good valve function.

\section{Discussion}

QAV is a rare congenital cardiac anomaly of the aortic valve, occurring in a prevalence of $0.008 \%$ and $0.043 \%$ in autopsy and echocardiographic studies, respectively [23]. In the literature, there is a discrete male predominance in relation to women, with a male-to-female ratio of 1.6:1. The diagnosis is most commonly performed from the 5th decade of life, with a mean age of 50.7 years, when these patients become clinically symptomatic. This fact is probably a consequence of progressive aortic valve injury resulting from this malformation, making valvular insufficiency more expressive after 5 decades, leading to the appearance (or exacerbation) of clinical manifestations consistent with aortic insufficiency. Due to the progressive character of aortic valve injury over the years, it is important to diagnose this malformation early, avoiding, through strict follow-up of these patients, the evolution to left ventricular disease (LV failure) [24].

Before the advent of Echocardiography, the diagnosis of QAV was usually made during cardiac surgeries or autopsies. The wide use of the echocardiogram, especially after 1980, facilitated the identification of QAV and, consequently, made this diagnosis more frequent. Konrad et al. in his review, found that $82 \%$ of cases of QAV were diagnosed after the 1980s [15]. Although TTE has an important role in detecting some congenital heart defects, it has a limitation in the identification of QAV due to its worse acoustic window for the study of the aortic valve. The TEE allows a higher image resolution of this valve (due to the great proximity between the esophagus and the left atrium and absence of bones or lung tissue), allowing a better definition of the morphology and degree of valvular dysfunction, as well as identifying the mechanisms responsible for its failure [18] [25]. The typical QAV image on the echocardiogram was described by Herman in 1984 and has since become pathognomonic of the disease. In the diastole, it shows the typical "cross" or "X" valve closure (Image 2) different from the characteristic " $Y$ " closure of the normal tricuspid aortic valve and, in systole, the rectangular opening, different from the typical triangular opening of the normal valve [26]. 


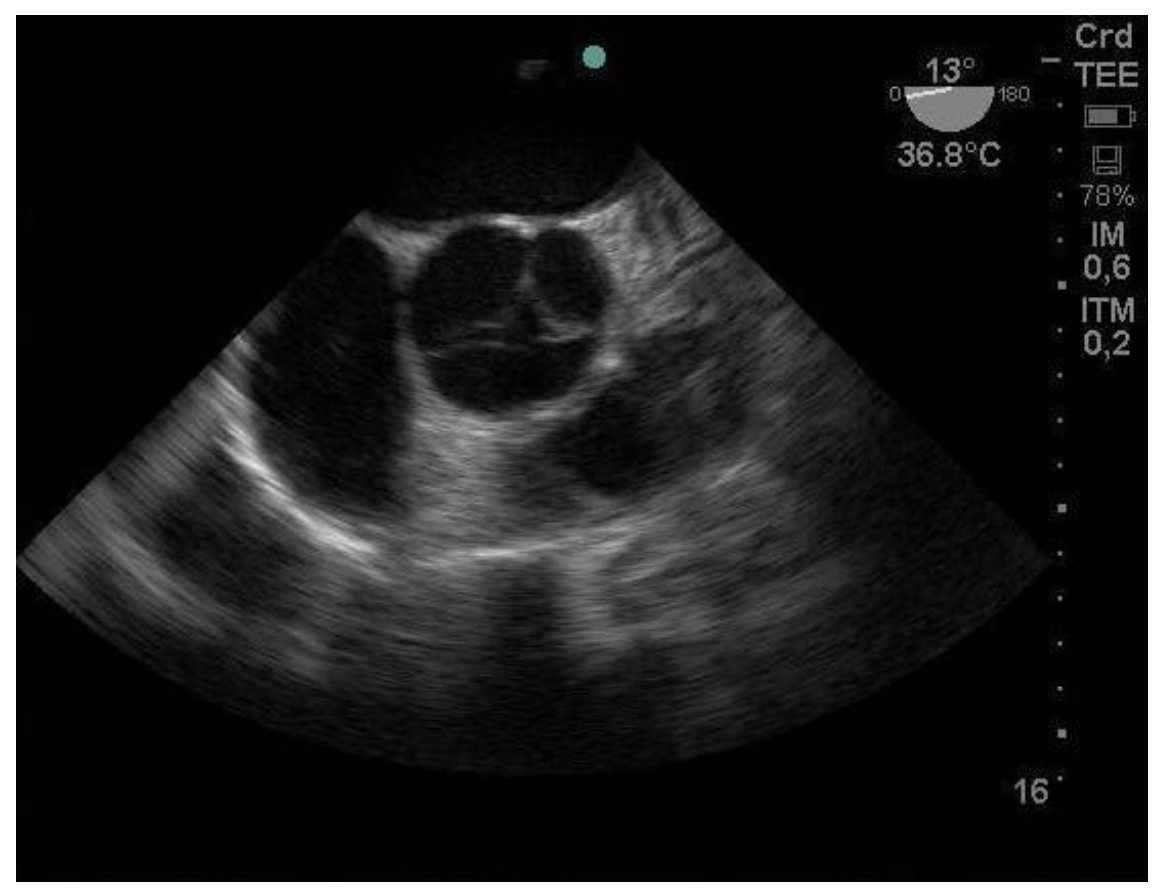

Image 2. Mid-esophageal cut short aortic valve obtained through intraoperative TEE exam, demonstrating a pathognomonic image of QAV ("X" or "cross" valve closure during diastole).

The pathophysiology of aortic insufficiency, presenting in $68 \%$ of the cases of patients with QAV, is still not very well established in the literature, and there are some theories that try to explain it: 1) valve injury due to unequal distribution of stress forces due to anatomical abnormalities of the cusps with subsequent development of fibrosis and calcification; 2) incomplete coaptation of the cusps by unequal sizes between them, preventing an ideal coaptation, which would be aggravated by fibrosis and posterior calcification; 3) dilation of the aortic root and prolapse of the cusps [24] [27]. In conclusion, it is believed that the development of thickening and valvar calcification, occurring throughout life, propitiated by the factors discussed above, are responsible for valvular insufficiency in QAV.

$\mathrm{QAV}$, for the most part, is an isolated anomaly, but occasionally it may be associated with other cardiac malformations. The most commonly found in the literature are those related to coronary artery ostia (single coronary ostia, anomalous positions or obstruction) anomalies, reported in $10 \%$ of the cases described [13]. Other cardiac malformations associated with QAV less frequently found were: ventricular septal defect, patent ductus arteriosus, and mitral valve malformation [14] [27]. In addition, there appears to be an increased risk of developing IE in patients with QAV, especially in those patients who have valves with anatomically unequal cusps because of increased flow disturbances. Thus, prophylaxis for IE should be instituted for all patients with QAV who will undergo procedures involving bacteremia [28].

The treatment of QAV will depend on the abnormalities found in the valve, 
referring to the degree of dysfunction and the degree of thickening and calcification. Surgical treatment is indicated for symptomatic patients and recommended for asymptomatic patients with evidence of left ventricular dysfunction (ejection fraction $<50 \%$ ) or enlargement of the left ventricle (diastolic diameter $>75 \mathrm{~mm}$ and systolic diameter $>55 \mathrm{~mm}$ ) which have not yet progressed to the stage of cardiac decompensation.

Since its introduction in clinical practice in the late 1980s, TEE has become one of the main diagnostic modalities in cardiology, as it guides anesthetic/surgical procedures and decreases morbidity and mortality in cardiac surgeries. The first guideline on perioperative TEE was published in 1999 by the Society of Cardiovascular Anesthesiologists/American Society of Echocardiography (SCA/ASE). In Brazil, we have the guidelines of the Brazilian Society of Cardiology (SBC) together with the Brazilian Society of Anesthesiology (SBA) on the use of intraoperative TEE. The levels of evidence and indications for the use of TEE in cardiac and noncardiac surgeries are shown in Table 1 [18] [29].

With level I of evidence, the TEE and its study with Doppler flowmetry used in the intraoperative (Image 3), demonstrated great importance in the surgical/anesthetic management of patients submitted to cardiac surgeries. TEE provides valuable qualitative and quantitative information contributes both to optimize hemodynamic monitor and to provide anatomical data that guides the surgical technique used, in addition to early identification of complications such as ischemia, air embolism or pericardial effusion [18] [29].

The pathophysiology of chronic aortic insufficiency involves the progressive

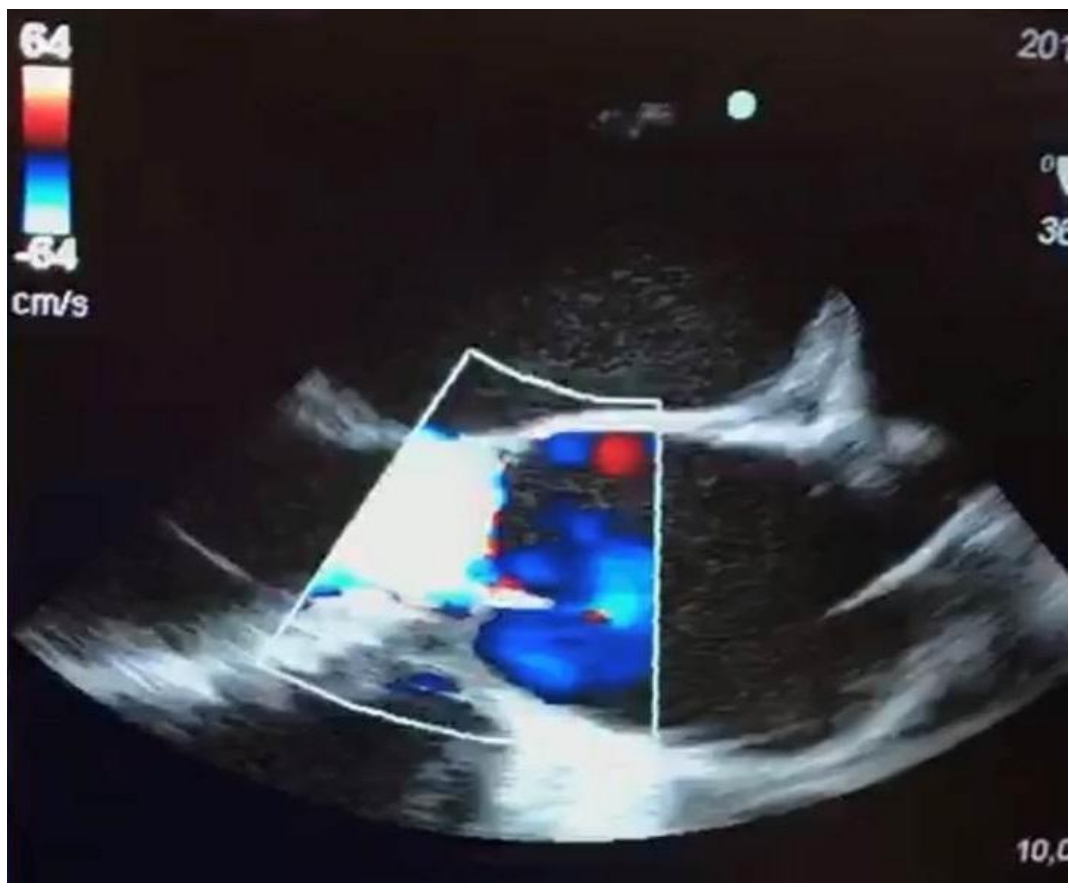

Image 3. Mid-long esophageal section of the aortic valve in an intraoperative TEE exam with color Doppler mode evidencing the presence of blood reflux through the incompetent AV. 
Table 1. Consensus on SBA/SBC Perioperative Transesophageal Echocardiography/Level of Evidence-Intraoperative Transesophageal Echocardiography.

\begin{tabular}{|c|c|}
\hline Recommendation & Class \\
\hline $\begin{array}{l}\text { Severe, acute, and persistent hemodynamic disorders, with doubtful ventricular function, } \\
\text { irresponsive to treatment }\end{array}$ & I \\
\hline $\begin{array}{l}\text { Valve lesion repair or surgical replacement, aortic disease, and hypertrophic } \\
\text { cardiomyopathy }\end{array}$ & I \\
\hline $\begin{array}{l}\text { Ventricular aneurysm, cardiac tumor removal, intracardiacthrombectomy, and } \\
\text { pulmonary embolectomy }\end{array}$ & I \\
\hline Congenital heart disease surgery with cardiopulmonary bypass & I \\
\hline Placement of intracardiac devices & I \\
\hline Assessment of localized or posterior pericardial effusion & I \\
\hline $\begin{array}{l}\text { Assessment of transcatheter procedures (interatrial communication closure, atrial } \\
\text { appendage occlusion, transcatheter valve procedures) }\end{array}$ & I \\
\hline $\begin{array}{l}\text { Myocardial function assessment after myocardial revascularization with or without } \\
\text { cardiopulmonary bypass }\end{array}$ & IIa \\
\hline Large non-cardiac surgery in high-risk patients & IIa \\
\hline
\end{tabular}

process of LV dilation and its eccentric hypertrophy as a consequence of the volume overload determined by retrograde blood flow (regurgitant systolic volume) into the LV during diastole through the incompetent aortic valve (AV).

$\mathrm{AV}$ insufficiency also determines low diastolic blood pressure. Both bradycardia and elevated diastolic systemic blood pressure increase regurgitant volume due, respectively, to longer diastole time and increased pressure gradient through AV (aortic diastolic pressure minus LV end-diastolic pressure), favoring blood flow retrograde. Thus, management of anesthesia in patients with chronic aortic regurgitation should include care that optimizes cardiac output by increasing the effective LV systolic volume and decreasing regurgitant volume. The heart rate should be maintained near the upper limits of normality ( 80 - 100 beats/min). Bradycardia and tachycardia should be avoided, as they may cause, respectively, regurgitant volume increase and contribution to myocardial ischemia. Cardiac output may still be improved by mild decreases in systemic vascular resistance and compensatory increase in cardiac preload (volume replacement should be performed with caution, since overexposure may easily result in pulmonary edema) [30].

Angina may occur even in the absence of coronary disease, as the myocardial oxygen demand is increased due to muscle hypertrophy and LV dilation, while the myocardial blood supply is reduced due to the low diastolic pressures in the aorta as a result of regurgitation. This fact, together with the risk of QAV association with coronary artery ostia anomalies, shows the importance of strict monitoring for myocardial ischemia in patients with valvular heart disease, especially when submitted to surgical stress.

TEE with flow Doppler study provides valuable information on the hemodynamic measures of the patient through data based on calculations that estimate 
cardiac pressures, volumes and measures of the heart, thus guiding the intraoperative anesthetic management. With the knowledge of estimated measures such as cardiac output, systolic/diastolic volume, regurgitant volume, ventricular ejection fraction and the evaluation of cardiac contractility, a better understanding of the present hemodynamic disorder can be obtained, and thus, to institute more specific therapeutic interventions and effective [18].

In addition, the TEE examination allows the intra/extracardiac and large anatomical analysis of the base, the detailed analysis of AV (its general morphology, number of valves, presence of calcifications and mobility) and the qualitative and quantitative analysis of the flows in color Doppler mode (Image 3) and pulsatile mode, thus allowing aortic regurgitation, estimating the size, mechanism and position of the regurgitant orifice [16] [18]. All these anatomical/hemodynamic measures can directly influence the decision of the surgical technique to be used, evidencing the importance of a detailed echocardiographic study in the region of the aortic root. The location of the coronary ostia (also well visualized in the TEE) should always be informed to the surgeons to prevent the accidental obstruction of these structures during the fixation of the prosthesis ring in valve replacement surgery, since, as previously explained, the association of QAV and the anomalous origin of Valsalva's sinus is not uncommon [15] [29].

\section{Conclusions}

Despite the low prevalence of QAV, its clinical significance comes from the fact that $68 \%$ of patients with this valvular heart disease have some degree of aortic insufficiency. The early diagnosis of this congenital malformation is of fundamental importance, since the lesion to the AV is progressive and may lead over the years to severe aortic insufficiency, LV failure and finally congestive heart failure.

The management of anesthesia in these patients should include care that optimizes cardiac output by increasing the effective LV systolic volume and decreasing regurgitant volume. Heart rate should be maintained near the upper limits of normality. Bradycardia and tachycardia should be avoided. Cardiac output may still be improved by mild decreases in systemic vascular resistance and compensatory caution increase in cardiac preload.

The Echocardiographic study has a recognized role in the diagnosis (supernumerary aortic valve detection) and in the follow-up of patients with QAV. In the last decades, the intraoperative use of TEE has been gaining prominence, especially in cardiac surgeries. This is because a complete examination during the intraoperative period not only characterizes the hemodynamic profile of the patient but also can lead to modifications in the surgical approach in up to $25 \%$ of the exams. Thus, based on the scientific evidence and direct impact on the final outcomes of cardiac surgeries (decrease in morbidity and mortality), the use of intraoperative TEE has been widely discussed by the Societies of Cardiology and Anesthesiology in order to define guidelines for its use. 


\section{References}

[1] Hurwitz, L.E. and Roberts WC. (1973) Quadricuspid Semilunar Valve. American Journal of Cardiology, 31, 623-626. https://doi.org/10.1016/0002-9149(73)90332-9

[2] Vali, Y., Rajendra, R. and Nishtar, S. (2010) A Previously Undescribed Type of Quadricuspid Aortic Valve: Type H. Journal of Heart Valve Disease, 19, 792-793.

[3] Nakamura, Y., Taniguchi, I., Saiki, M., Morimoto, K. and Yamaga, T. (2001) Quadricuspid Aortic Valve Associated with Aortic Stenosis and Regurgitation. Japanese Journal of Thoracic and Cardiovascular Surgery, 49, 714-716. https://doi.org/10.1007/BF02913511

[4] Tutarel, O. and Westhoff-Bleck, M. (2008) Functional Status of the Quadricuspid Aortic Valve/an Uncommon Coincidence of Congenital Quadricuspid Aortic Valve Accompanied by Hypertrophic Obstructive Cardiomyopathy. The Anatolian Journal of Cardiology, 8, 86.

[5] Sousa, L., Pinto, F., Nogueira, G., Kaku, S. and Antunes, A.M. (2001) Quadricuspid Aortic Valve and Atrial Septal Defect. Revista Portuguesa de Cardiologia, 20, 329-330.

[6] Demirkol, S., Balta, S., Arslan, Z., Unlu, M., Kucuk, U. and Iyisoy, A. (2013) Association of Quadricuspid Aortic Valve and Ventricular Septal Defect in a Patient Who Had Undergone Atrial Septal Defect Surgery. Polish Heart Journal, 71, 546. https://doi.org/10.5603/KP.2013.0112

[7] Seol, S.H., Kim, U., Cho, H.J., Kim, D.K., Kim, D.I. and Kim, D.S. (2010) Quadricuspid Aortic Valve with Patent Ductus Arteriosus. Texas Heart Institute Journal, 37, 726-727.

[8] Suzuki, Y., Daitoku, K., Minakawa, M., Fukui, K. and Fukuda, I. (2006) Congenital Quadricuspid Aortic Valve with Tetralogy of Fallot and Pulmonary Atresia. Japanese Journal of Thoracic and Cardiovascular Surgery, 54, 44-46.

https://doi.org/10.1007/BF02743785

[9] Egred, M., Patel, J.C. and Metcalfe, M.J. (2005) Sinus of Valsalva Fistula with Quadricuspid Aortic Valve, a First Reported Association. International Journal of Cardiology, 101, 151-152. https://doi.org/10.1016/j.ijcard.2004.01.029

[10] Iglesias, A., Oliver, J., Muñoz, J.E. and Nuñez, L. (1981) Quadricuspid Aortic Valve Associated with Fibromuscular Subaortic Stenosis and Aortic Regurgitation Treated by Conservative Surgery. CHEST Journal, 80, 327-328. https://doi.org/10.1378/chest.80.3.327

[11] Irisawa, T., Yoshiya, K., Yokosawa, T., Iwamatsu, T., Arai, K. and Aoki, T. (1993) A Case of Quadricuspid Aortic Valve Associated with Mitral Regurgitation. Japanese Journal of Thoracic Surgery, 46, 618-621.

[12] Sakamoto, Y., Saitoh, F., Ohnishi, K., Kurosawa, H. and Takakura, H. (1994) A Case of Quadricuspid Aortic Valve Associated with Mitral Insufficiency. Japanese Journal of Thoracic and Cardiovascular Surgery, 42, 1235-1237.

[13] Janssens, U., Klues, H.G. and Hanrath, P. (1997) Congenital Quadricuspid Aortic Valve Anomaly Associated with Hypertrophic Non-Obstructive Cardiomyopathy: A Case Report and Review of the Literature. Heart, 78, 83-87. https://doi.org/10.1136/hrt.78.1.83

[14] Erdmenger, J., Vázquez-Antona, C., Becerra, R., Romero, A., Roldan, J., Buendía, A., et al. (2005) Quadricuspid Aortic Valve in a Patient with D-Transposition of the Great Arteries. Archivos De Cardiologia De Mexico, 75, 460-462.

[15] Konrad, R., et al. (2009) Quadricuspid Aortic Valve: A Complete Review. Brazilian 
Journal of Cardiovascular Imaging and Echocardiograph, 22, 39-52.

[16] Tutarel, O. (2004) The Quadricuspid Aortic Valve: A Comprehensive Review. Journal of Heart Valve Disease, 13, 534-537

[17] Timperley, J., Milner, R., Marshall, A.J., Marshall, J.A. and Gilbert, T.J. (2002) Quadricuspid Aortic Valves. Clinical Cardiology, 25, 548-552. https://doi.org/10.1002/clc.4950251203

[18] Lim, D.S., Dent, J.M., Gutgesell, H.P., Matherne, P. and Kron, I.L. (2007) Transesophageal Echocardiographic Guidance for Surgical Repair of Aortic Insufficiency in Congenital Heart Disease. Journal of the American Society of Echocardiography, 20, 1080-1085. https://doi.org/10.1016/j.echo.2007.01.031

[19] Tsang, M.Y., Abudiab, M.M., Ammash, N.M., Naqvi, T.Z., Edwards, W.D., Nkomo, V.T., et al. (2016) Quadricuspid Aortic Valve: Characteristics, Associated Structural Cardiovascular Abnormalities, and Clinical Outcomes. Circulation, 133, 312-319.

[20] Mecozzi, G., Pratali, S., Milano, A., Nardi, C. and Bortolotti, U. (2003) Severe Quadricuspid Aortic Valve Stenosis after Mediastinal Irradiation. Journal of Thoracic and Cardiovascular Surgery, 126, 1198-1199.

https://doi.org/10.1016/S0022-5223(03)00368-4

[21] Idrees, J.J., Roselli, E.E., Arafat, A., Johnston, D.R., Svensson, L.G., Sabik 3rd, J.F., et al. (2015) Outcomes after Repair or Replacement of Dysfunctional Quadricuspid Aortic Valve. Journal of Thoracic and Cardiovascular Surgery, 150, 79-82. https://doi.org/10.1016/j.jtcvs.2015.03.019

[22] Pirundini, P.A., Balaguer, J.M., Lilly, K.J., Gorsuch, W.B., Taft, M.B., Cohn, L.H., et al. (2006) Replacement of the Quadricuspid Aortic Valve: Strategy to Avoid Complete Heart Block. Annals of Thoracic Surgery, 81, 2306-2308. https://doi.org/10.1016/j.athoracsur.2005.08.022

[23] Ketty, S., Quintavalle, E. and Ambrosio, G. (2015) Quadricuspid Aortic Valve: A Case Report and Review of the Literature. Journal of Cardiovascular Echography, 25, 72-76.

[24] Di Pino, A., Gitto, P., Silvia, A. and Bianca, I. (2008) Congenital Quadricuspid Aortic Valve in Children. Cardiology in the Young, 18, 324-327. https://doi.org/10.1017/S1047951108002205

[25] Godefroid, O., Colles, P., Vercauteren, S., Louagie, Y. and Marchandise, B. (2006) Quadricuspid Aortic Valve: A Rare Etiology of Aortic Regurgitation. European Journal of Echocardiography, 7, 168-170. https://doi.org/10.1016/j.euje.2005.03.011

[26] Herman, R.L., Cohen, I.S., Glaser, K., Newcomb 3rd, E.W. (1984) Diagnosis of Incompetent Quadricuspid Aortic Valve by Two-Dimensional Echocardiography. American Journal of Cardiology, 53, 972. https://doi.org/10.1016/0002-9149(84)90546-0

[27] Marek, B., et al. (2006) Transesophageal Echocardiography of the Quadricuspid Aortic Valve. Echo Rounds in Evolution: Anesthesia \& Analgesia, 103, 1414-1415.

[28] Tarasoutchi, F., Montera, M.W., Grinberg, M., Barbosa, M.R., Piñeiro, D.J., Sánchez, C.R.M. and Barbosa, M.M. (2011) Diretriz Brasileira de Valvopatias-SBC 2011/I Diretriz Interamericana de Valvopatias-SIAC 2011. Arquivos Brasileiros de Cardiologia, 97, 1-6. https://doi.org/10.1590/S0066-782X2011002000001

[29] Salgado-Filho, M.F., et al. (2018) Consenso sobre Ecocardiografia Transesofágica Perioperatória da Sociedade Brasileira de Anestesiologia (ETTI/SBA) e do Departamento de Imagem Cardiovascular da Sociedade Brasileira de Cardiologia [Consensus on Perioperative Transesophageal Echocardiography of the Brazilian Society of Anesthesiology and the Department of Cardiovascular Image of the 
Brazilian Society of Cardiology]. Brazilian Journal of Anesthesiology, 68, 1-32.

[30] Edward, M.G., Mikhail, M.S. and Murray, M.J. (2006) Clinical Anesthesiology. Lange Medical Books/McGraw Hill Medical Pub. Division, New York. 